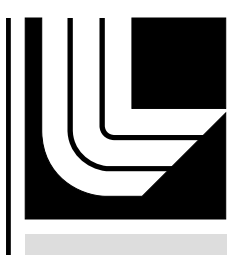

LAW RENCE LIVERMORE N A TIO N A L LABORATORY

Non-LTE Radiation Transport in High Radiation Plasmas

H. A. Scott

January 14, 2005

High Energy Density Physics 
This document was prepared as an account of work sponsored by an agency of the United States Government. Neither the United States Government nor the University of California nor any of their employees, makes any warranty, express or implied, or assumes any legal liability or responsibility for the accuracy, completeness, or usefulness of any information, apparatus, product, or process disclosed, or represents that its use would not infringe privately owned rights. Reference herein to any specific commercial product, process, or service by trade name, trademark, manufacturer, or otherwise, does not necessarily constitute or imply its endorsement, recommendation, or favoring by the United States Government or the University of California. The views and opinions of authors expressed herein do not necessarily state or reflect those of the United States Government or the University of California, and shall not be used for advertising or product endorsement purposes. 


\title{
Non-LTE Radiation Transport in High Radiation Plasmas
}

\author{
Howard A. Scott ${ }^{*}$ \\ Lawrence Livermore National Laboratory, P.O. Box 808, L-18, Livermore, CA 94551, USA
}

\begin{abstract}
A primary goal of numerical radiation transport is obtaining a self-consistent solution for both the radiation field and plasma properties. Obtaining such a solution requires consideration of the coupling between the radiation and the plasma. The different characteristics of this coupling for continuum and line radiation have resulted in two separate sub-disciplines of radiation transport with distinct emphases and computational techniques. LTE radiation transfer focuses on energy transport and exchange through broadband radiation, primarily affecting temperature and ionization balance. Non-LTE line transfer focuses on narrowband radiation and the response of individual level populations, primarily affecting spectral properties. Many high energy density applications, particularly those with high-Z materials, incorporate characteristics of both these regimes. Applications with large radiation fields including strong line components require a non-LTE broadband treatment of energy transport and exchange.

We discuss these issues and present a radiation transport treatment which combines features of both types of approaches by explicitly incorporating the dependence of material properties on both temperature and radiation fields. The additional terms generated by the radiation dependence do not change the character of the system of equations and can easily be added to a numerical transport implementation. A numerical example from a Z-pinch application demonstrates that this method improves both the stability and convergence of the calculations. The information needed to characterize the material response to radiation is closely related to that used by the Linear Response Matrix (LRM) approach to near-LTE simulation, and we investigate the use of the LRM for these calculations.
\end{abstract}

Keywords: Non-LTE; Radiation Transport

*E-mail address: hascott@llnl.gov

\section{Introduction}

Radiation transport methods have been applied to a wide variety of physical systems over the last several decades. Solution techniques are now sufficiently well developed to allow routine usage of multidimensional simulations in the fields of astrophysics and high energy density physics. In particular, applications in two regimes have been well studied in these fields. Energy transport by radiation in high temperature plasmas in local thermodynamic equilibrium (LTE) is an important, if not dominant, phenomenon in applications such as stellar interiors and inertial confinement fusion (ICF), in which the radiation interacts with matter and transports energy over a wide range of frequencies. The importance of this broadband radiation transport has led to a great deal of work over the last few decades, resulting in the development of very effective numerical schemes [1].

The situation is similar for transport of line radiation in non-LTE applications such as stellar atmospheres and plasma spectroscopy. In the regime of interest for these applications, a very narrow frequency range of radiation interacts with the matter, altering the populations of atomic energy levels, ultimately affecting the spectrum emitted by the matter. Effective numerical algorithms now allow the solution of extremely large and complex systems [2,3].

Despite the successes achieved in these two fields, much work remains in the development of efficient, robust and general solution methods. Non-LTE materials in high radiation fields, common in high energy 
density physics applications, respond strongly to radiation in both their energetic and spectral characteristics. The motivation behind this paper is the extension of a class of commonly used numerical radiation transport methods to handle such applications.

In the following section, we consider the non-LTE energetics of radiation interacting with matter, determining the relationship between energy density and temperature. The generalization of the LTE relationship separates the dependence of the energy density on the temperature from the direct effect of radiative interactions. A numerical example then identifies the regime in which the radiation spectrum can significantly alter the material response.

The equations and solution methods considered in this paper are presented in Sec. 3. The set of equations used for broadband radiation transport and material energetics is given in Sec. 3a, along with a straightforward solution scheme. Similarly, Sec. 3b presents the equations used for line radiation transport, along with a very common solution technique. For each of these cases, we briefly discuss those aspects of the physical situation and numerical techniques that contribute to the effectiveness of the solution methods. The emphasis here is on the coupling of the radiation to the material, and on the self-consistent solution for the radiation field and material properties. The discussion leads naturally to an extension of the broadband algorithm which explicitly incorporates the dependence of material properties on radiation. The extended algorithm is presented and discussed in Sec. 3c, along with a low-density approximation used to calculate the additional material response terms.

Both the original solution scheme for the broadband equations and the extended algorithm use linearization to incorporate material response information. A majority of the discussion and conclusions contained in this paper should apply to any solution scheme that shares this characteristic. Solution techniques that do not explicitly incorporate response information, e.g. those depending on a robust nonlinear solver, will not benefit directly, but the insights afforded by this work should still be valuable.

Since our interest here is in handling the coupling between radiation and matter, we do not explicitly consider the numerical solution of the radiative transport equation itself. We will actually use a diffusion operator rather than a true transport operator for numerical work, with the assumption that this does not alter the character of the radiation-matter coupling. Sec. 4 presents results from a test application based upon a dynamic hohlraum, for both the standard broadband algorithm and for the extended algorithm.

The additional terms required by the extended algorithm are very expensive to compute, and add to the cost of the already-expensive non-LTE calculations. In Sec. 5, we address the possibility of using tabular information for the material properties and responses, which would greatly decrease the cost of the nonLTE simulations. The linear response matrix (LRM) approach to near-LTE simulations [4] tabulates response information similar to that required by the extended algorithm, and we consider a generalization of this approach that would be suitable for our purposes.

All numerical results presented in this paper use material properties calculated self-consistently with the radiation field by a collisional-radiative model [5]. The electronic structure and transition rates are calculated using a modified screened-hydrogenic atomic model very similar to that described in [6].

\section{Non-LTE Energetics}

For matter that is not in LTE, describing the response to radiation is more complicated than for the corresponding LTE case. We consider the relationship between the material energy density $E_{m}$, the material properties and the radiation field:

$$
E_{m}=\frac{3}{2}\left(n_{e}+n_{i}\right) k T+E_{\text {int }}\left(T, J_{v}, t\right)
$$

Here, $n_{e}\left(n_{i}\right)$ is the number density of the free electrons (ions), which are assumed to have a thermal distribution corresponding to the material temperature $T$. $E_{\text {int }}$ is the material internal energy, which depends not only on the temperature and density, but also on the radiation field, denoted by $J_{v}$, and on the time $t$. 
For the remainder of this paper, we ignore the density dependence as unimportant to the discussion and focus on the temperature and radiation. We also adopt a single-temperature description of the material for simplicity of exposition.

For material in LTE, the internal energy depends only on temperature, and the rate of change in material energy density and temperature are related through the specific heat (at constant density) $C_{V}$ :

$$
\frac{d E_{m}}{d t}=c_{V}^{L T E} \frac{d T}{d t}, c_{V}^{L T E}=\left(\frac{\partial E_{m}}{\partial T}\right)_{J_{v}=B_{v}}
$$

Implicit in this formulation is the assumption that either radiative interactions are completely unimportant or that the extant radiation also has a thermal distribution, i.e. $J_{v}=B_{v}$, where $B_{v}$ is the Planck distribution. In the more general non-LTE formulation, the rate of change of material energy density is comprised of three different types of terms:

$$
\frac{d E_{m}}{d t}=\left(\frac{\partial E_{m}}{\partial T}\right)_{J_{v}} \frac{\partial T}{\partial t}+\sum_{v}\left(\frac{\partial E_{m}}{\partial J_{V}}\right)_{T} \frac{\partial J_{v}}{\partial t}+\left(\frac{\partial E_{m}}{\partial t}\right)_{J_{v}, T}
$$

The first term on the RHS of Eq. (3) describes the response of the material energy density to a change in temperature, but with fixed radiation densities, while the second term describes the material response to a change in radiation at fixed temperature. The coefficient of the first term plays the part of the non-LTE specific heat, which is related to the LTE specific heat by

$$
c_{V}^{L T E}=c_{V}^{\text {NLTE }}+\sum_{v}\left(\frac{\partial E_{m}}{\partial J_{v}}\right)_{T} \frac{\partial B_{V}}{\partial T}, c_{V}^{\text {NLTE }}=\left(\frac{\partial E_{m}}{\partial T}\right)_{J_{V}}
$$

The last term on the RHS of Eq. (3) arises from evolution of the material at fixed temperature and radiation, and acts as a source or sink of energy. This term can be quite important in following the thermal evolution of matter at very low densities and temperatures, but for the remainder of this discussion we assume this term is negligible and do not consider it further.

Non-LTE effects will become significant at densities low enough for important radiative transition rates to become comparable to the corresponding collisional rates. A numerical example illustrates the relative importance of the temperature and radiative responses to the specific heat. For this example, we calculate the specific heat of a Lu plasma at three different densities. Figures 1a - 1c show the specific heat as a function of temperature for ion number densities of $10^{18}, 10^{20}$ and $10^{22} \mathrm{~cm}^{-3}$, respectively. In each figure, the thick solid line gives the LTE specific heat, ${c_{V}}^{\text {LTE }}$, while the thin solid line gives the non-LTE specific heat $c_{V}{ }^{N L T E}$ evaluated assuming a Planckian radiation field at the given temperature, and the thin dashed line gives $c_{V}{ }^{N L T E}$ evaluated assuming no radiation field. The dotted line gives the specific heat obtained by evaluating Eq. (4) using approximate values for the derivatives with respect to $J_{v}$. This lowdensity approximation (the "diagonal” approximation) will be explained in Sec. 3.3.

At the highest of the three densities, LTE is a good approximation and the specific heat varies little with the radiation. As the density decreases, the difference between $c_{V}{ }^{L T E}$ and $c_{V}{ }^{N L T E}$ increases, and it becomes apparent that the material radiative response dominates the temperature response. Regardless of the other considerations in this paper, use of the LTE specific heat at low densities in the presence of nonPlanckian radiation fields will not describe the material energetics correctly.

\section{Radiation Transport}

In the first two parts of this section, we consider salient features of numerical radiation transport algorithms as employed in common applications for both broadband (continuum) and line radiation. For both these cases, we list the basic set of equations to be solved, i.e. the radiative transfer equation together with the appropriate material equation(s), and briefly discuss certain aspects of their solution. The emphasis here is on those aspects critical to constructing a self-consistent solution method with reasonable convergence properties. Although these subsections repeat information broadly available in the literature, 
they provide the components for the last subsection, which proposes an extension of the broadband algorithm that incorporates features from both cases.

\subsection{Broadband radiation transport}

The system of equations describing energy transport by broadband radiation is comprised of the radiation transport equation

$$
\frac{1}{c} \frac{\partial I_{v}}{\partial t}+\vec{\Omega} \bullet \nabla I_{v}=-\left(\alpha_{v} I_{v}-\eta_{v}\right)=-\alpha_{v}\left(I_{v}-S_{v}\right)
$$

and the material energy equation

$$
\frac{\partial E}{\partial t}=4 \pi \int \alpha_{v}\left(J_{v}-S_{v}\right) d v+Q
$$

where $I_{v}$ is the specific intensity at frequency $v, \alpha_{v}$ and $\eta_{v}$ are the absorption coefficient and emissivity, $Q$ represents other energy sources, $J_{v}$ is the angle-averaged intensity

$$
J_{v}=\frac{1}{4 \pi} \int I_{v} d \Omega
$$

and $S_{v}=\eta_{v} / \alpha_{v}$ is the source function. In LTE, the source function is the Planck function, $B_{v}$ and is a function of temperature only.

A common method of solving this non-linear set of equations is to discretize in time and linearize about the current temperature $T^{0}$. Applying these operations to Eq. (6) gives

$$
c_{T}\left(T-T^{0}\right)=\Delta t \int \alpha_{v}\left(J_{v}-S_{v}{ }^{0}\right) d v+Q \Delta t
$$

where

$$
c_{T}=c_{V}+\Delta t \int \alpha_{v} \frac{\partial S_{V}}{\partial T} d v
$$

acts as a specific heat for the total system of matter and radiation. Eq. (8) can be analytically combined with the linearized and discretized version of Eq. (5), resulting in

$$
\begin{aligned}
\frac{1}{c} \frac{I_{v}-I_{v}{ }^{0}}{\Delta t}+\vec{\Omega} \bullet \nabla I_{v} & =-\alpha_{v}\left(I_{v}-S_{v}{ }^{0}\right)+\alpha_{v} \frac{\partial S_{v}}{\partial T}\left(T-T^{0}\right) \\
& =-\alpha_{v}\left(I_{v}-S_{v}{ }^{0}\right)+\frac{\Delta t}{c_{T}} \alpha_{v} \frac{\partial S_{v}}{\partial T} \int \alpha_{v}\left(J_{v}-S_{v}{ }^{0}\right) d v+Q \frac{\Delta t}{c_{T}} \alpha_{v} \frac{\partial S_{v}}{\partial T}
\end{aligned}
$$

We have assumed a fully-implicit time discretization, since applications usually require at least a partially-implicit treatment for stability. The superscript "0" denotes values at the beginning of the time interval. This treatment can be, and in LTE often is, generalized to an iterative procedure to converge the nonlinear dependence of $S_{v}$ on the temperature.

The integral terms in Eq. (10) couple together intensities for all angles and all frequencies. Our primary interest here is the coupling of the different radiation frequencies to the material, so we simplify the equations further by eliminating the angular dimensions. Replacing the transport operator with a diffusion operator through the substitutions

$$
I_{v} \leftarrow J_{v}, \quad \vec{\Omega} \bullet \nabla I_{v} \leftarrow-\nabla \bullet D_{v} \nabla J_{v}
$$

in Eq. (10), where $D_{v}=c / 3 \alpha_{v}$ is the diffusion coefficient, simplifies both the analysis while leaving the essential aspects of coupling between radiation and material unchanged.

The resulting multigroup radiation diffusion equations converge very slowly under a straightforward iterative procedure, but can be made to converge quickly upon application of multifrequency-grey acceleration [7]. As discussed in this paper, a stability analysis reveals that without acceleration, short wavelength modes can be marginally convergent. The grey acceleration operator provides much faster convergence, eliminating the error in these modes by transporting a correction term with a spectral shape 
determined by the absorption coefficient spectrum. The correction accounts for redistribution of radiation into optically thin high frequencies, where it can propagate freely.

The efficiency of grey acceleration does depend upon the spectrum of the absorption coefficient. If the absorption coefficient falls off too quickly with frequency, the acceleration degrades or fails. However, physically realistic continuum opacities tend to fall of as $\sim v^{-3}$ at high frequencies, slowly enough for grey acceleration to be effective.

\subsection{Line radiation transport}

In this case, the radiation transport equation (5) is combined with the atomic kinetics rate equation

$$
\frac{d \mathbf{y}}{d t}=\mathbf{A y}
$$

where the vector $\mathbf{y}$ represents the population densities of the atomic levels and $\mathbf{A}$ is the rate matrix. The total rate $A_{i j}$ connecting two atomic levels $i$ and $j$ includes collisional and radiative transitions, both discrete (bound-bound) and continuous (free-bound). The populations respond to the radiation through the effects on the transition rates.

The prototypical example of this type of system is the steady-state two-level atom [8], consisting of two levels connected by a single discrete radiative transition and a collisional transition. The source function for such a system, obtained under the approximation that the width of the spectral line is very narrow (and assuming complete redistribution), has the form:

$$
S_{v}=\frac{2 h v_{i j}{ }^{3}}{c^{2}} \frac{y_{j}}{\left[y_{i}-\left(g_{j} / g_{i}\right) y_{j}\right]}=\frac{2 h v_{i j}{ }^{3}}{c^{2}} S_{i j}(\bar{J})
$$

where $v_{i j}$ is the transition frequency and $g_{i}$ is the statistical weight of level $i$. The frequency-independent source function $S_{i j}$ depends on the radiation field only through the angle-integrated, frequency-averaged quantity

$$
\bar{J}=\int_{0}^{\infty} J_{v} \phi(v) d v
$$

which enters into the transition rate, where $\phi$ is the line profile function. For this simple system, $S_{i j}$ has the form

$$
S_{i j}(\bar{J})=(1-\varepsilon) \bar{J}+\varepsilon B_{i j}
$$

where $B_{i j}$ is the non-dimensional Planck function at the transition energy. $\varepsilon$ is determined by the ratio of the collisional and spontaneous radiative transition rates and can be very small for a strong radiative transition. The more general source function accounting for all overlapping radiative transitions (including free-free transitions) is somewhat more complicated, but in the low-density regime it remains true that for a frequency corresponding to a strong transition $i \rightarrow j$, the dominant radiative dependence will be on the corresponding $\bar{J}$.

As with broadband radiation transport, there are a variety of techniques designed to produce selfconsistent converged solutions with reasonable convergence rates. Over the last couple decades, the most versatile and economical methods have been based on the concept of approximate operators [9]. Rather than invert the full transport operator, these methods invert an approximation to the full operator, using the inverse in an iterative procedure to obtain the full solution. The closer the approximate operator is to the full operator, the faster the procedure converges. There are many ways to choose an approximate operator, and several dimensions to work in, but the near-universal choice for multidimensional problems is to use the (spatial) diagonal of the full operator [10]. This is trivial to invert, being a scalar, and relatively simple to form. These tradeoffs compensate for a somewhat slower convergence rate.

The diagonal approximate operator contains contributions from all frequencies contributing to the line radiation, but in contrast to the broadband acceleration operator, it contains only local information about the material response. This does not contradict the broadband analysis, as the absorption spectrum for a 
spectral line violates the conditions for grey acceleration to be effective. The troublesome high frequency modes do not exist in line radiation and it suffices to handle the frequency-frequency coupling locally.

\subsection{Combined broadband + line radiation}

The solution techniques described in the last two subsections work quite well in their respective regimes, but this success does not carry over between regimes. For instance, an approximate operator technique applied to broadband radiation with a standard absorption spectrum converges very slowly. If a high-frequency cutoff is added to the absorption spectrum, mimicking a line spectrum, the approximate operator converges quickly but grey acceleration becomes ineffective.

The considerations of the previous two subsections suggest that a simple combined approach might be effective in situations where both broadband radiation and line radiation are important. We retain the structure of the broadband treatment, including a grey acceleration step to treat high-frequency modes, but add the local dependence of the material properties on the radiation spectrum by linearizing the source function in the radiation field as well in the temperature:

$$
S_{v}=S_{v}\left(T, J_{v^{\prime}}\right) \approx S_{v}\left(T^{0}, J_{v^{\prime}}{ }^{0}\right)+\frac{\partial S_{v}}{\partial T}\left(T-T^{0}\right)+\sum_{v^{\prime}} \frac{\partial S_{v}}{\partial J_{v^{\prime}}}\left(J_{v^{\prime}}-J_{v^{\prime}}{ }^{0}\right)
$$

This linearization of the source term is a straightforward generalization along the lines of the discussion of energetics in Sec. 2. In the regime where the radiation spectrum significantly influences the energetics, it is reasonable to expect a similarly significant dependence in the source function. The considerations of the previous two subsections provide cause for optimism that this simple extension will produce a stable convergent solution to radiation-dominated non-LTE problems. Sec. 4 presents a numerical application of this method demonstrating support for this viewpoint.

The new response terms $\partial S_{v} / \partial J_{v^{\prime}}$ involve all frequencies. These terms do not introduce any new complications into the solution method, but computing all the additional derivatives is extremely expensive. In the low-density regime where we expect strong line radiation to dominate the radiative response, we can make the additional approximation that each bound-bound radiative transition $R_{i j}$ responds to radiation of a single frequency. Implicit in this approximation is the assumption that each strong line is contained within a single frequency bin, and we make no attempt to resolve any of the lines. Under these conditions, we can easily calculate the required derivatives from the atomic kinetics equations. We refer to this as the "diagonal" approximation, as it uses only the diagonal terms from the complete response matrix. Figs. (1a) - (1c) demonstrate that this approximation indeed does very well at low densities, but poorly near LTE.

Extending the solution method described in Sec. 3a to include the new response terms is straightforward. Linearizing the equations about the current temperature and radiation spectrum, using the diagonal approximation, produces

$$
\begin{aligned}
& C_{T}\left(T-T^{0}\right)=\Delta t \int \alpha_{v}\left(J_{v}-S_{v}{ }^{0}-\frac{\partial S_{v}}{\partial J_{v}}\left(J_{v}-J_{v}{ }^{0}\right)\right) d v-\sum_{v}\left(\frac{\partial E_{m}}{\partial J_{v}}\right)_{T}\left(J_{v}-J_{v}{ }^{0}\right)+Q \Delta t \\
& \frac{1}{c} \frac{J_{v}-J_{v}{ }^{0}}{\Delta t}-\nabla \bullet D_{v} \nabla J_{v}=-\alpha_{v}\left(J_{v}-S_{v}{ }^{0}\right)+\alpha_{v}\left[\frac{\partial S_{v}}{\partial T}\left(T-T^{0}\right)+\frac{\partial S_{v}}{\partial J_{v}}\left(J_{v}-J_{v}{ }^{0}\right)\right]
\end{aligned}
$$

where $c_{T}$ is defined as in Eq. (9) using the non-LTE specific heat. Eqs. (17) and (18) retain the same multigroup structure as before, and may be solved in the same manner.

A numerical implementation of the broadband equations can be extended in a very simple manner. Most of the changes in the implementation are captured by the substitutions:

$$
\alpha_{v} J_{v} \leftarrow \tilde{\alpha}_{v} J_{v}=\alpha_{v} J_{v}\left(1-\frac{\partial S_{v}}{\partial J_{v}}\right), S_{v}{ }^{0} \leftarrow \tilde{S}_{v}{ }^{0}=S_{v}{ }^{0}-\frac{\partial S_{v}}{\partial J_{v}} J_{v}{ }^{0}
$$

In the absence of the diagonal approximation, these changes include the obvious sums over frequencies. Besides these substitutions, only a couple additional terms remain to be handled separately. 
One significant change in solution procedure from standard LTE practice is necessitated by the nonLTE nature of the material properties. The source function is no longer a Planckian and need not have a simple form, so any iterative procedure that attempts to produce a self-consistent solution will necessarily entail iterating the atomic kinetics equations as well. This would be prohibitively expensive in most cases, although Sec. 5 discusses one approach towards a tabular solution.

A related issue has to do with the grey acceleration step, which uses Planckian weights and is intended for use within the iterative procedure. However, an alternative procedure is to directly solve Eq. (10) for all frequencies simultaneously. A reduction procedure makes this quite economical in one dimension. Test cases, including the examples presented in the following section, have shown that using a single grey acceleration step produces nearly the same results as the direct solution procedure. Grey acceleration remains remarkably effective for non-LTE problems as well as for LTE problems.

\section{Test Case: Radiation-driven Cylinder}

As a test of the extended transport algorithm, we consider a case that is based upon a Z-pinch dynamic hohlraum experiment [11]. The specifications are very similar to those used in [12] to model tungsten liners. The chosen configuration, illustrated in Fig. 2, consists of a hollow cylinder composed of uniform density Lu. The cylinder is illuminated from the interior by a blackbody radiation source of temperature $250 \mathrm{eV}$, with vacuum on the exterior. The inner surface of the cylinder has a radius of $\mathrm{r}_{0}=0.16 \mathrm{~cm}$ and the outer surface has a radius of $r_{3}=0.36 \mathrm{~cm}$. The radii $r_{1}$ and $r_{2}$, equally spaced between $r_{0}$ and $r_{3}$, are identified for purposes of displaying results. The goal is to calculate the self-consistent temperature and radiation distribution throughout the cylinder.

Although this is a static geometry and we seek the steady-state solution, we perform time-dependent calculations with each time step corresponding to a single atomic kinetics evaluation followed by a single application of either the broadband or extended transport algorithm, using one grey acceleration correction.

We consider three different densities for the cylinder. At the lowest of these, with number density $N_{i}=$ $10^{18} \mathrm{~cm}^{-3}$, the cylinder is optically thick only at frequencies corresponding to strong line transitions. The highest density, $N_{i}=10^{20} \mathrm{~cm}^{-3}$, corresponding to the middle density used for the specific heat evaluations in Sec. 2, is not yet in LTE, but has moderate to high optical depths over most of the frequency range. Fig. 3 shows the optical depth along a radial line between the inner and outer radii for these two cases, evaluated from fully converged NLTE solutions, as well as the optical depth for the lower density case, evaluated from an LTE solution.

Figs. $4 \mathrm{a}$ and $4 \mathrm{~b}$ show the radiation intensity, $J_{v}$, at the inner and outer radii for the low-density and the high-density cases, respectively. The Planck function appropriate to the material temperature is included for reference. The low-density case is very far from LTE, and the intensity spectra largely reflect the driving radiation, with some modifications due to strong line transitions. The high-density case is reasonably close to LTE, although a number of non-Planckian features are visible in the intensity spectra. The source function, $S_{n}$, provides a better indication of the non-LTE nature of these cases, as shown in Figs. $5 \mathrm{a}$ and $5 \mathrm{~b}$. Again, the appropriate Planck functions are included for reference.

A more interesting measure of the non-LTE nature of these cases is given in Figs. 6a and 6b. These figures display the diagonal of the source response function, $\partial S_{v} / \partial J_{v}$, as a function of frequency, at both the inner and outer radii for the low-density and the high-density cases. This quantity is strictly zero in LTE, as the source function is independent of the radiation field. For the two-level atom, this quantity reaches a maximum value of one when collisional rates are negligible. A large value in this context also indicates highly radiation-dominated transitions. A value too close to one can cause problems in the numerical implementation, as evidenced in Eq. (19), so we restrict the maximum value of this quantity to 0.95. This limit is also reflected in the figures. It is evident that various narrow spectral ranges of the source function are very strongly dependent on the radiation field, indicating that these spectral ranges are 
dominated by strong line transitions. We also define an average value of this quantity by integrating over the spectrum, weighted by the radiation intensity:

$$
\left\langle\frac{\partial S}{\partial J}\right\rangle=\int \frac{\partial S_{v}}{\partial J_{v}} J_{v} d v / \int J_{v} d v
$$

For the low-density case, the average value is about 0.47 over the entire spatial domain, indicating a very strong dependence on the radiation field. For the high-density case, the average value varies from 0.27 0.37 . Since the diagonal approximation is not accurate for this case, these values are not reliable, but may still indicate a degree of sensitivity to the radiation field.

The converged material and radiation temperature profiles for the low-density case, for both non-LTE and LTE calculations, are given in Fig. 7a. The radiation temperature is insensitive to the material treatment and changes very slowly with radius. The material temperature, however, differs considerably for these two treatments.

The difference in behavior of the broadband and extended algorithms, as shown in Fig. 7b, is quite dramatic. The solutions were obtained through time-dependent evolution, starting from a uniform material temperature of $200 \mathrm{eV}$. The timesteps were initially very small, gradually increasing while attempting to keep temperature changes small within each timestep. Fig. $7 \mathrm{~b}$ displays temperature histories for four equally spaced points from the inner radius to the outer radius. The histories generated by the extended algorithm are smooth and well behaved. The broadband algorithm, however, evolves in the wrong direction early in time and quickly goes unstable. Stabilizing the evolution requires timesteps small enough for an explicit algorithm.

Figs. 8a and 8b give the corresponding results for the mid-density case, with $N_{i}=10^{19} \mathrm{~cm}^{-3}$. The spatial temperature profiles still differ significantly between the non-LTE and LTE solutions, particularly near the outer boundary of the cylinder. The extended algorithm again performs well, smoothly evolving to the steady-state solution. The broadband algorithm experiences some difficulty, experiencing a mild instability at low temperatures, but stabilizes and smoothly evolves to late times. However, slow evolution continues at very late times and the solution does not reach steady state.

The results for the high-density case are given in Figs. 9a and 9b. Here, the non-LTE and LTE spatial temperature profiles are indistinguishable, although the material and radiation temperatures still differ noticeably near the boundaries. For this case, the broadband algorithm performs well, evolving smoothly to the steady-state solution. The extended algorithm does not perform quite as well at early times and goes unstable at late times. This behavior is almost certainly due to the failure of the diagonal approximation at this high density.

The extended algorithm does expand the range of conditions for which this type of radiation transport algorithm can be successfully applied. It improves both the stability and the convergence of the solution, at least when the diagonal approximation to the response function is appropriate. Unfortunately, it is not necessarily clear when this approximation is valid. Similarly, in mildly non-LTE situations it may not be apparent when the broadband algorithm becomes inaccurate. In addition, calculating the diagonal response function increases the cost of expensive non-LTE simulations, although these additional calculations could undoubtedly be optimized.

Developing criteria for determining when to apply the diagonal response function would be one path towards applying the extended algorithm as a general-purpose algorithm. Alternatively, using the full response function would bypass this difficulty, but at a high computational cost since all (or many) crossderivatives would also be required. To avoid this expense, we are investigating the use of tabulated material properties, including full response functions, using a technique closely related to the linear response matrix method for near-LTE conditions. 


\section{Linear Response Matrix}

The linear response matrix (LRM) is a symmetric matrix $R_{v v^{\prime}}$ describing the change in energy absorption and emission at frequency $v$ due to the deviation of the radiation field from a Planckian at frequency $v$ ' [4].

$$
R_{v v^{\prime}}=4 \pi \frac{\partial}{\partial J_{v^{\prime}}}\left(\eta_{v}-\alpha_{v} B_{v}\right)^{\partial B_{v^{\prime}}} / \partial T_{e}
$$

The symmetry of the LRM follows from the general principle of detailed balance [13]. An equivalent definition is given by the relationship

$$
4 \pi\left(\eta_{v}-\alpha_{v} B_{v}\right)=\int R_{v v^{\prime}} \frac{J_{v^{\prime}}-B_{v^{\prime}}}{\partial B_{v^{\prime}} / \partial T_{e}} d v^{\prime}
$$

where the LHS of Eq. (22) is recognized as the quantity entering into the material energy equation. The computational utility of the LRM derives from the framework it provides for calculating near-LTE radiation transport problems using only tabular information. For our purposes, we require the individual derivatives from Eq. (21), instead of just the combination.

The extent to which the linear description assumed by the LRM remains valid under non-LTE conditions will be the subject of a future discussion. For our present purpose, we note that the components of the LRM, are the response quantities required by the extended transport algorithm, including all crossderivatives. In this context, we view the LRM as a tabulation of these derivatives. The questions to be addressed here are whether the derivatives evaluated at LTE can be used advantageously in the extended algorithm, and whether the tabular approach can be extended to situations far from LTE.

In practice, the tabulated derivatives must be used carefully, with limits placed on both the intensity deviations $\left(J_{v}-B_{v}\right)$ used to calculate corrections, and on the corrections themselves. With these constraints, the method works quite well for the high-density test case of Sec. 4, reproducing the results of Fig. (9a). However, since an LTE treatment does just as well, the derivatives have no significant effect here. For the mid-density case, the resulting temperature profiles are shown in Fig. (10) along with the LTE and nonLTE results. Using the tabulated derivatives reproduces some of the features of the non-LTE solution, but overall the results are slight worse than the strictly LTE treatment. Similar results are obtained at lower densities. The cause of this behavior is under investigation.

One possible failure of the LRM approach is overstepping the linear regime. Extending the reach of the tabular approach will require tabulating information not only at LTE, but for non-LTE conditions as well. We are now experimenting with evaluating the required quantities at points characterized by separate material and radiation temperatures. The additional parameter used in the tables is the ratio of the radiation temperature to the material temperature. Early results from this work are encouraging, but much work remains to be done.

\section{Summary}

Solving the radiation transport equation also involves evaluating material properties that can depend on the radiation field. In LTE, the material properties depend only on the material temperature, and the computational focus lies in calculating energy transport by broadband radiation and energy exchange between the material and radiation. In non-LTE, the material properties can depend directly on the radiation field, and the coupling between the material and radiation takes on a different character, which is reflected in the computational algorithms. In this paper, we have demonstrated an extended version of a broadband radiation transport algorithm that combines features of both types of transport schemes. The extended algorithm successfully handles applications with strong broadband and line radiation. Incorporating line radiation features into the algorithm improves both the stability and convergence of 
simulations with strong radiation fields, as demonstrated by an example based on a Z-pinch dynamic hohlraum.

The numerical implementation of the extended algorithm requires additional information about the response of the material properties to radiation. The full set of response information is prohibitively expensive to calculate inline with the radiation transport, but a low-density approximation using only the diagonal of the response matrix, only modestly increases the computational cost. Preliminary investigations into the use of tabulated material information, including the full response matrix, have been only slightly encouraging. However, a successful approach of this type would not only permit routine use of the extended algorithm, but would greatly speed up broadband non-LTE calculations. This will be a topic for future research.

\section{Acknowledgments}

This work was performed under the auspices of the U.S. Department of Energy, by the University of California, Lawrence Livermore National Laboratory under contract W-7405-ENG-48. 


\section{References}

[1] Adams ML, Larsen EW. Fast iterative methods for discrete-ordinates particle transport calculations. Prog in Nucl Energy2002;40:3-159.

[2] Kalkofen W, editor. Methods in radiative transfer. Cambridge: Cambridge University Press, 1984.

[3] Molisch AF, Oehry, BP. Radiation trapping in atomic vapours. Oxford: Oxford University Press, 1998.

[4] More RM, Kato T, Libby SB, Faussurier G. Atomic processes in near-equilibrium plasmas. J Quant Spectrosc Radiat Transfer 2001;71:505-518.

[5] Scott HA. Cretin - a radiative transfer capability for laboratory plasmas. J Quant Spectrosc Radiat Transfer 2001;71:689-701.

[6] Chung HK, Chen MH, Morgan WL, Lee RW. FLYCHK: Simple but generalized population kinetics and spectral model. J Quant Spectrosc Radiat Transfer, this issue.

[7] Morel JE, Larsen EW, Matzen MK. A synthetic acceleration scheme for radiative diffusion calculations. J Quant Spectrosc Radiat Transfer 1985;34:243-261.

[8] Mihalas D. Stellar atmospheres. San Francisco: W.H. Freeman and Co., 1978.

[9] Kalkofen W. Survey of operator perturbation methods. In: Kalkofen W, editor. Numerical radiative transfer. Cambridge: Cambridge University Press, 1987. p. 23-34.

[10] Olson GA, Auer LH, Buchler JR. A rapidly convergent iterative solution of the non-LTE line radiation transfer problem. J Quant Spectrosc Radiat Transfer 1986;35:431-442.

[11] Matzen MK. Z pinches as intense x-ray sources for high-energy density physics applications. Phys Plasmas 1997;4:1519-1527.

[12] Novikov VG, Zakharov SV. Modeling of nonequilibrium radiating tungsten liners. J Quant Spectrosc Radiat Transfer 2003;81:339-354.

[13] Faussurier G, More RM. Non-local thermodynamic equilibrium response matrix. J Quant Spectrosc Radiat Transfer 2003;76:269-288. 


\section{Figure Captions}

Fig. 1a Specific heat per ion as a function of temperature for a Lu plasma of number density $10^{18} \mathrm{~cm}^{-3}$, in units $\mathrm{eV} / \mathrm{eV}$. The thick solid line gives the LTE specific heat, the thin solid line gives the non-LTE specific heat for a Planckian radiation field $\left(T_{r}=T_{e}\right.$, where $T_{e}$ is the material temperature and $T_{r}$ is the radiation temperature), and the thin dashed line gives the non-LTE specific heat with no radiation field $\left(T_{r}=0\right)$. The dotted line gives the specific heat obtained from Eq. (4) using the diagonal approximation.

Fig. 1b Same as Fig. 1a for a number density of $10^{20} \mathrm{~cm}^{-3}$.

Fig. 1c Same as Fig. 1a for a number density of $10^{22} \mathrm{~cm}^{-3}$.

Fig. 2 Diagram of geometry for example application, consisting of an annular cylinder comprised of uniform density Lu between radii $r_{0}=0.16 \mathrm{~cm}$ and $r_{3}=0.36 \mathrm{~cm}$, illuminated at $r=r_{0}$ by a $250 \mathrm{eV}$ Planckian radiation source.

Fig. 3 Optical depth as a function of photon energy along a radial line between $r_{0}$ and $r_{3}$. The upper solid curve is for a number density of $10^{20} \mathrm{~cm}^{-3}$ and the lower solid curve is for a density of $10^{18} \mathrm{~cm}^{-3}$. The dotted curve is for a density of $10^{18} \mathrm{~cm}^{-3}$, assuming LTE.

Fig. 4a Radiation intensity (thick line), $J_{v}$, and Planck function (thin line), $B_{v}$, at $\mathrm{r}=\mathrm{r}_{0}$ (solid lines) and $\mathrm{r}=$ $\mathrm{r}_{3}$ (dotted lines) for a number density of $10^{18} \mathrm{~cm}^{-3}$.

Fig. 4b Same as Fig. 4a for a number density of $10^{20} \mathrm{~cm}^{-3}$.

Fig. 5a Source function (thick line), $\mathrm{S}_{v}$, and Planck function (thin line), $B_{v}$, at $\mathrm{r}=\mathrm{r}_{0}$ (solid lines) and $\mathrm{r}=\mathrm{r}_{3}$ (dotted lines) for a number density of $10^{18} \mathrm{~cm}^{-3}$.

Fig. 5b Same as Fig. 5a for a number density of $10^{20} \mathrm{~cm}^{-3}$.

Fig. 6a Diagonal source response function, $\partial S_{v} / \partial J_{v}$, at $\mathrm{r}=\mathrm{r}_{0}$ (solid lines) and $\mathrm{r}=\mathrm{r}_{3}$ (dotted lines) for a number density of $10^{18} \mathrm{~cm}^{-3}$.

Fig. $6 \mathrm{~b}$ Same as Fig. 5a for a number density of $10^{20} \mathrm{~cm}^{-3}$.

Fig. 7a Final material temperature (solid curves) and radiation temperature (dashed curves) profiles for a number density of $10^{18} \mathrm{~cm}^{-3}$. The heavy curves correspond to a converged non-LTE calculation and the light curves correspond to an LTE calculation.

Fig. 7b Material temperature as a function of time at positions $r=r_{0}, r_{1}, r_{2}$ and $r_{3}$ for a number density of $10^{18} \mathrm{~cm}^{-3}$. The solid lines correspond to a non-LTE calculation using the extended algorithm as described in the text, while the dashed lines correspond to a non-LTE calculation using the broadband algorithm.

Fig. 8a Same as Fig. 6a for a number density of $10^{19} \mathrm{~cm}^{-3}$.

Fig. $8 \mathrm{~b}$ Same as Fig. $6 \mathrm{~b}$ for a number density of $10^{19} \mathrm{~cm}^{-3}$.

Fig. 9a Same as Fig. 6a for a number density of $10^{20} \mathrm{~cm}^{-3}$. The non-LTE results were obtained without the intensity derivatives.

Fig. 9b Same as Fig. $6 \mathrm{~b}$ for a number density of $10^{20} \mathrm{~cm}^{-3}$. 
Fig. 10 Final material temperature for a number density of $10^{19} \mathrm{~cm}^{-3}$. The heavy curve corresponds to a converged non-LTE calculation, the light solid curve corresponds to an LTE calculation, and the light dashed curve corresponds to a calculation using tabulated LRM information. 


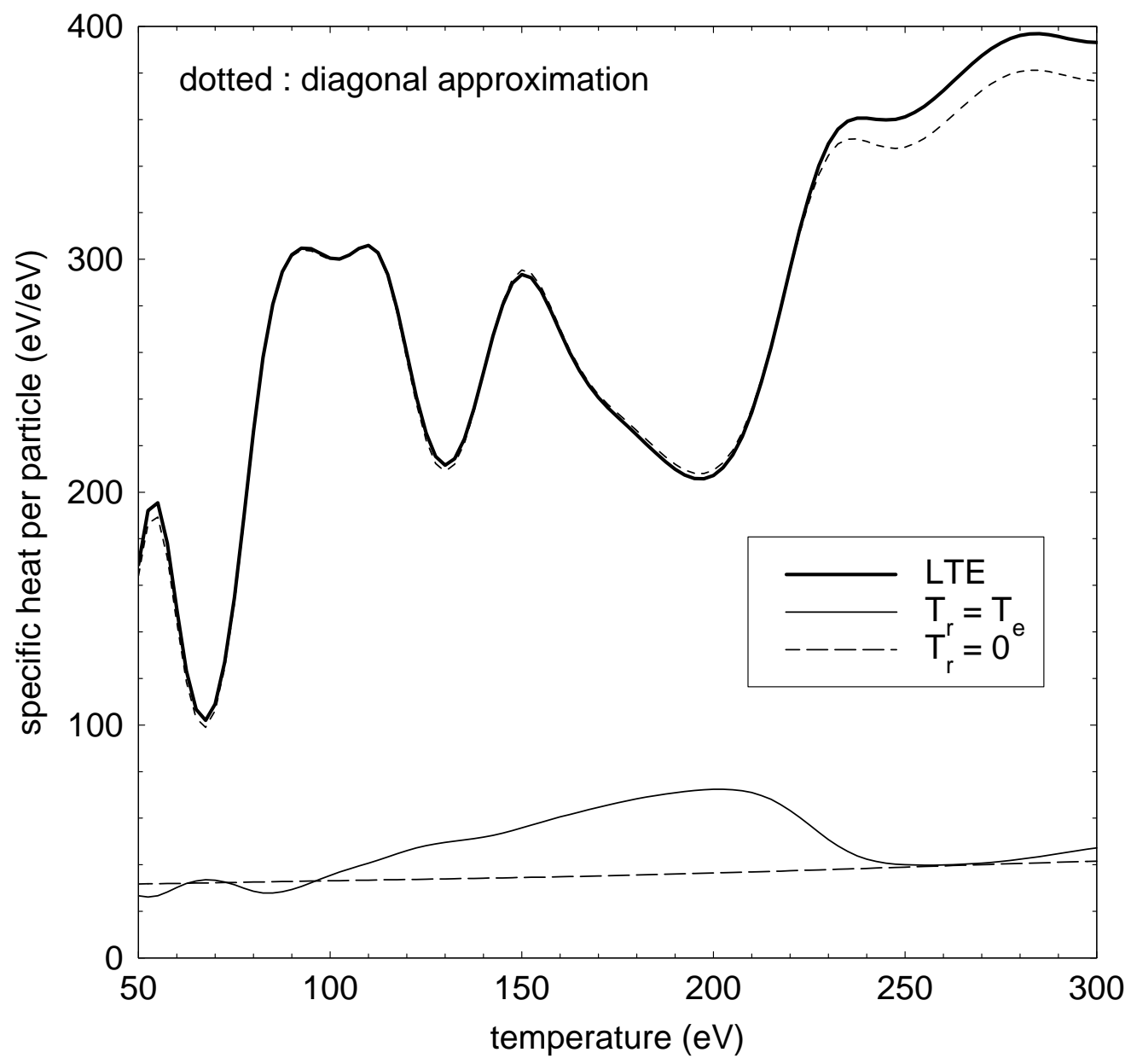

Figure 1a 


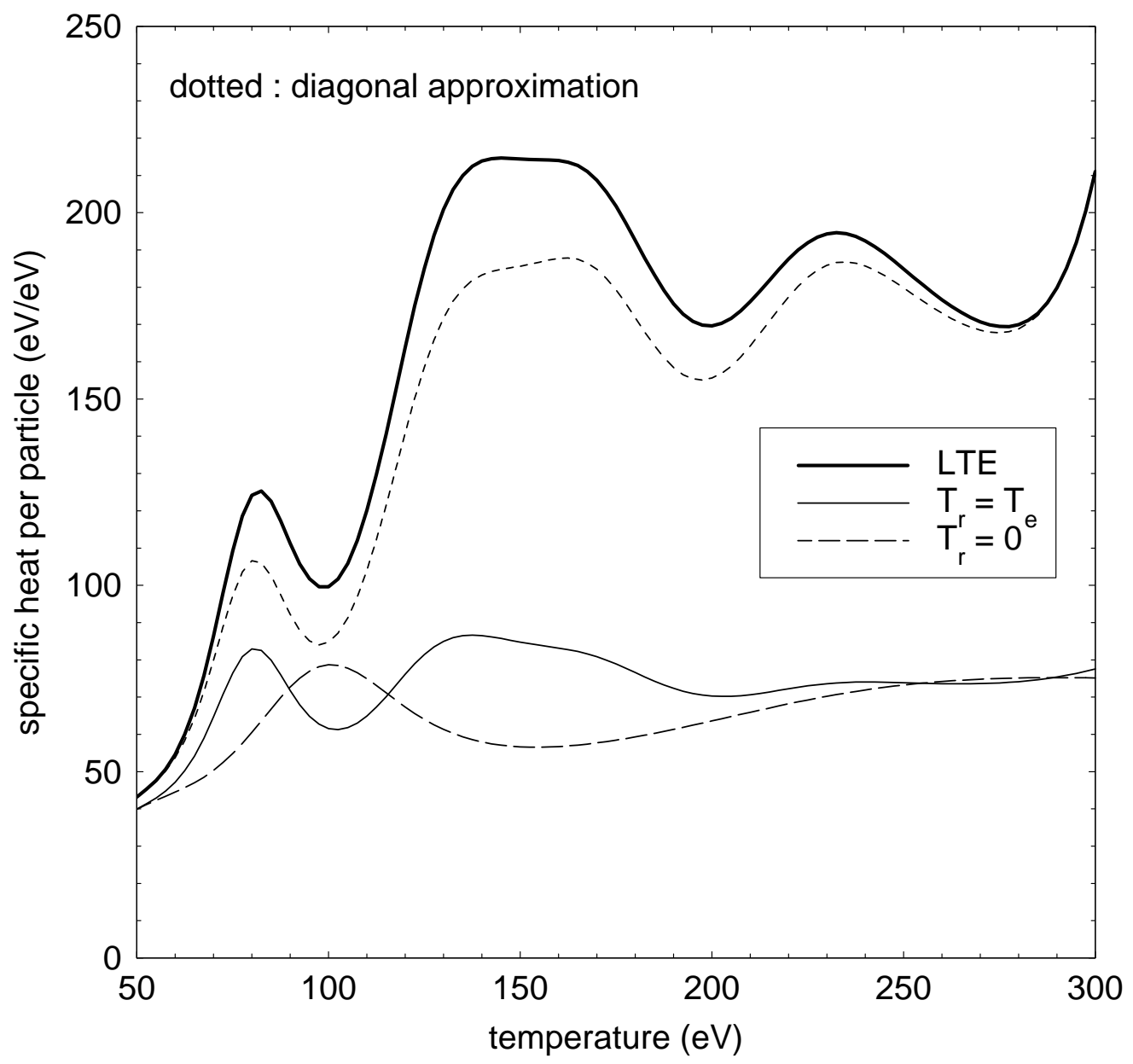

Figure 1b 


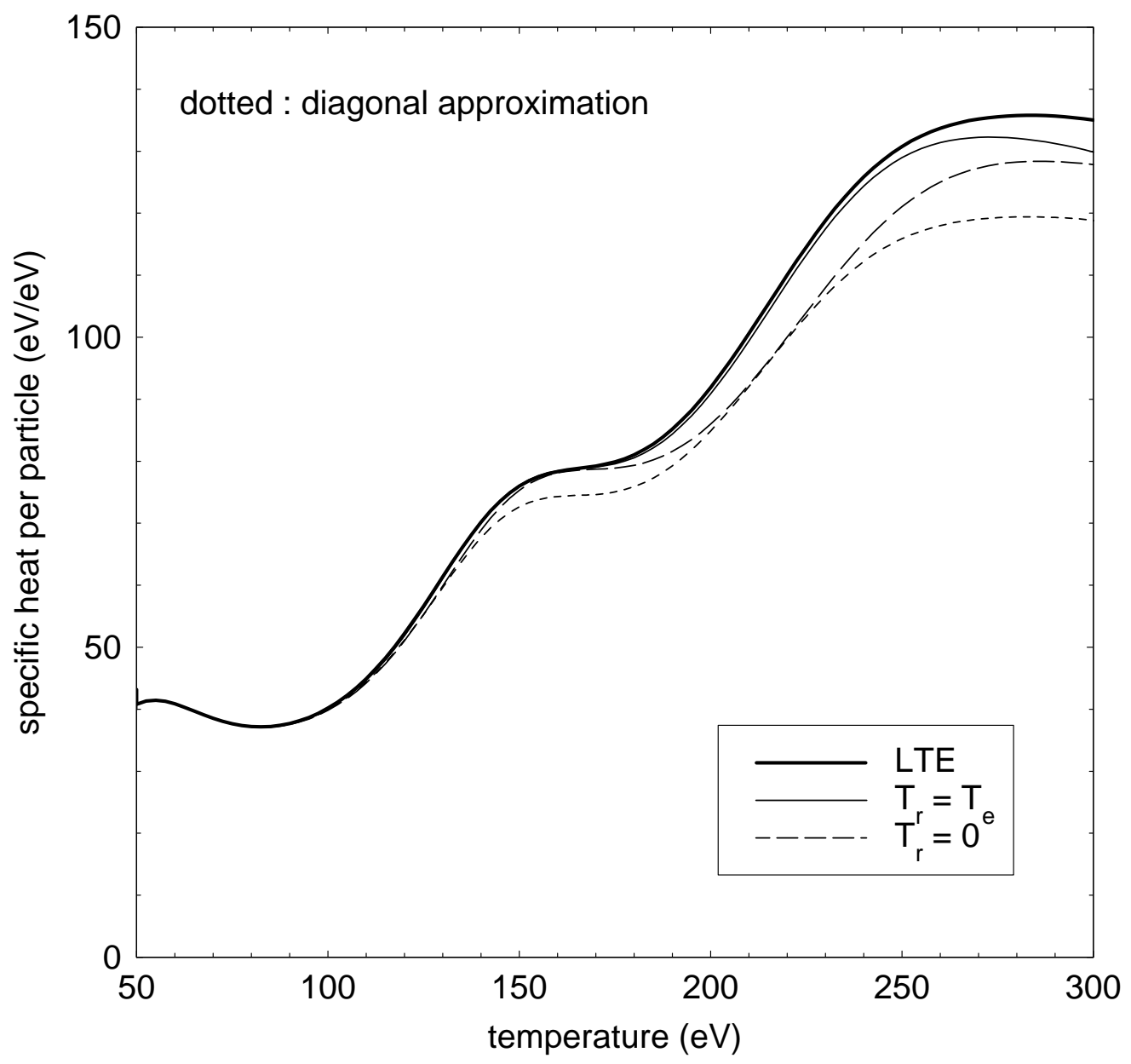

Figure 1c 


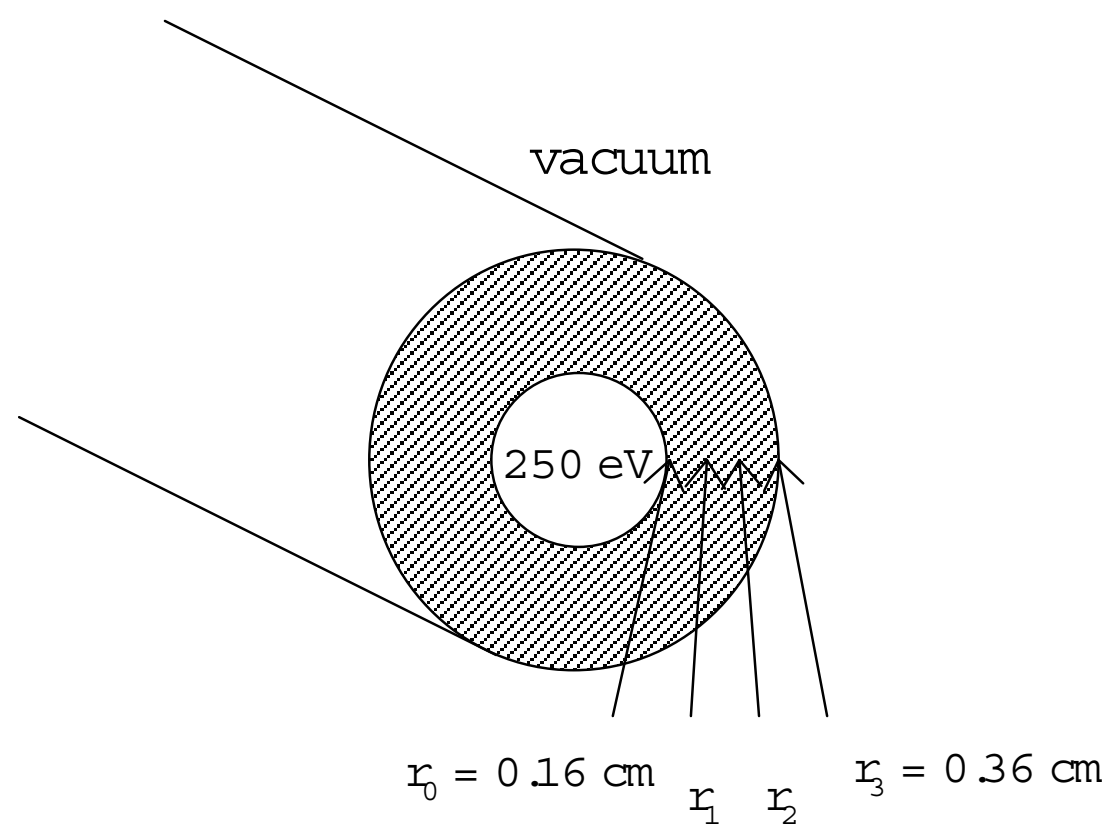

Figure 2 


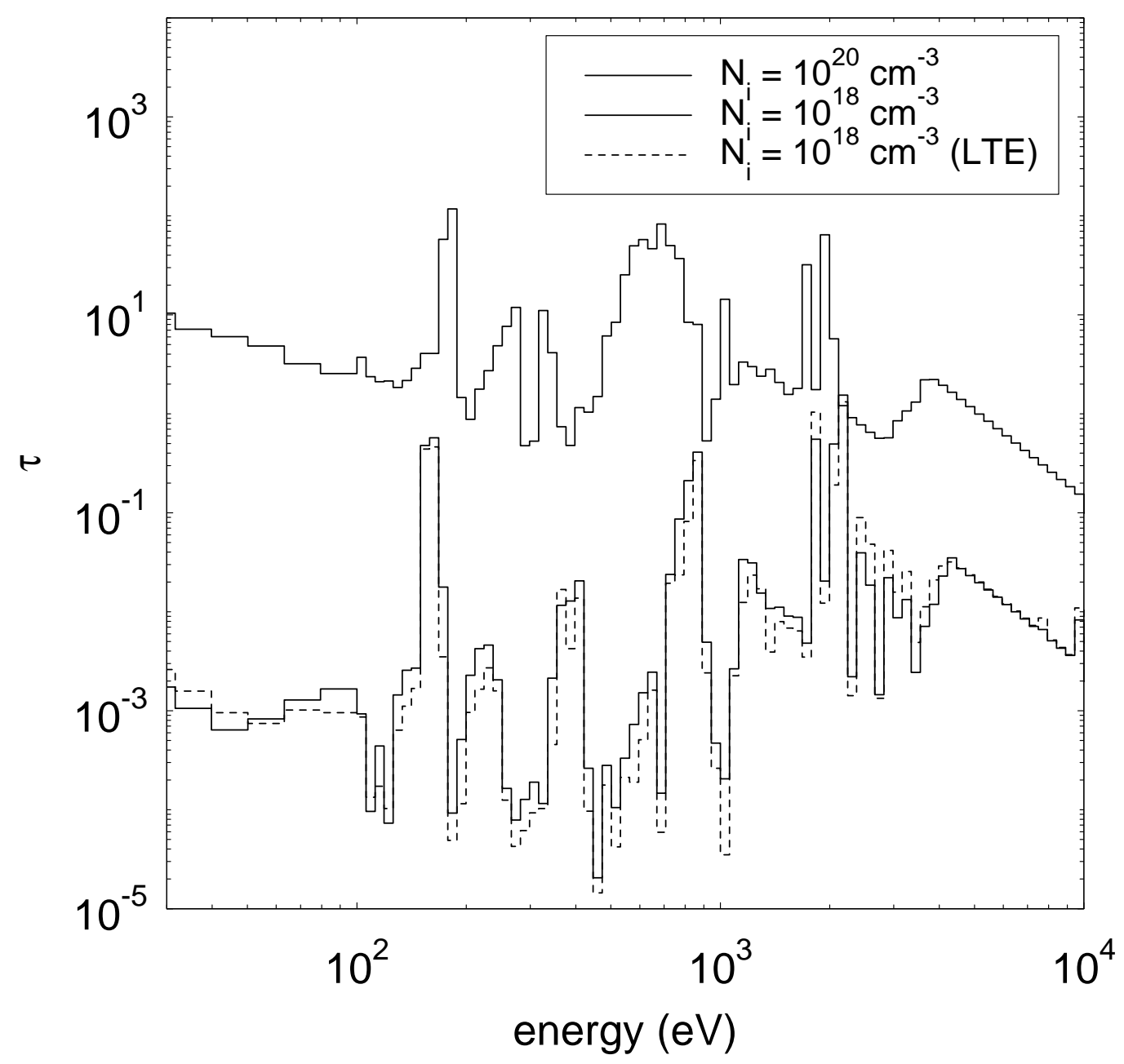

Figure 3 


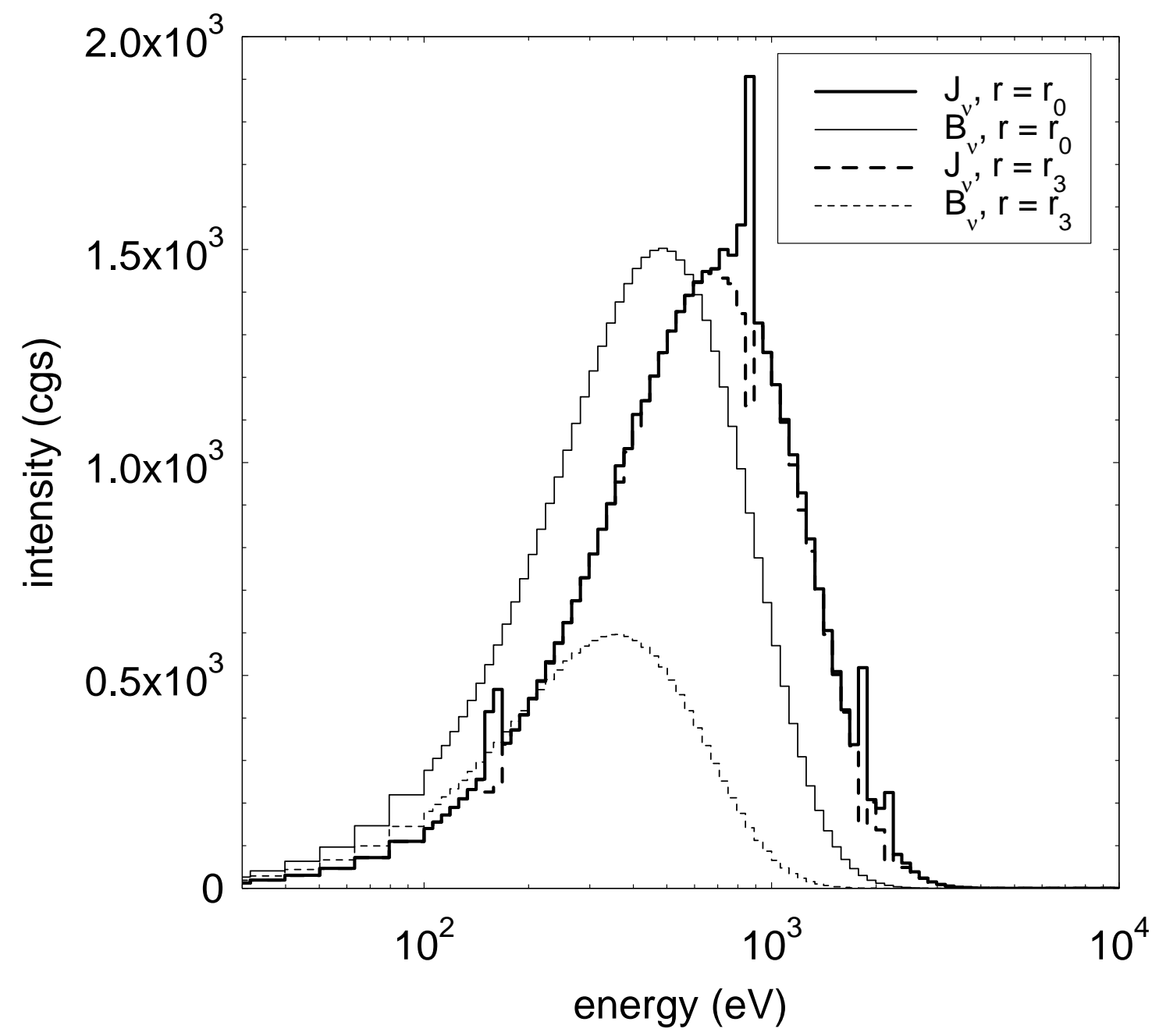

Figure 4a 


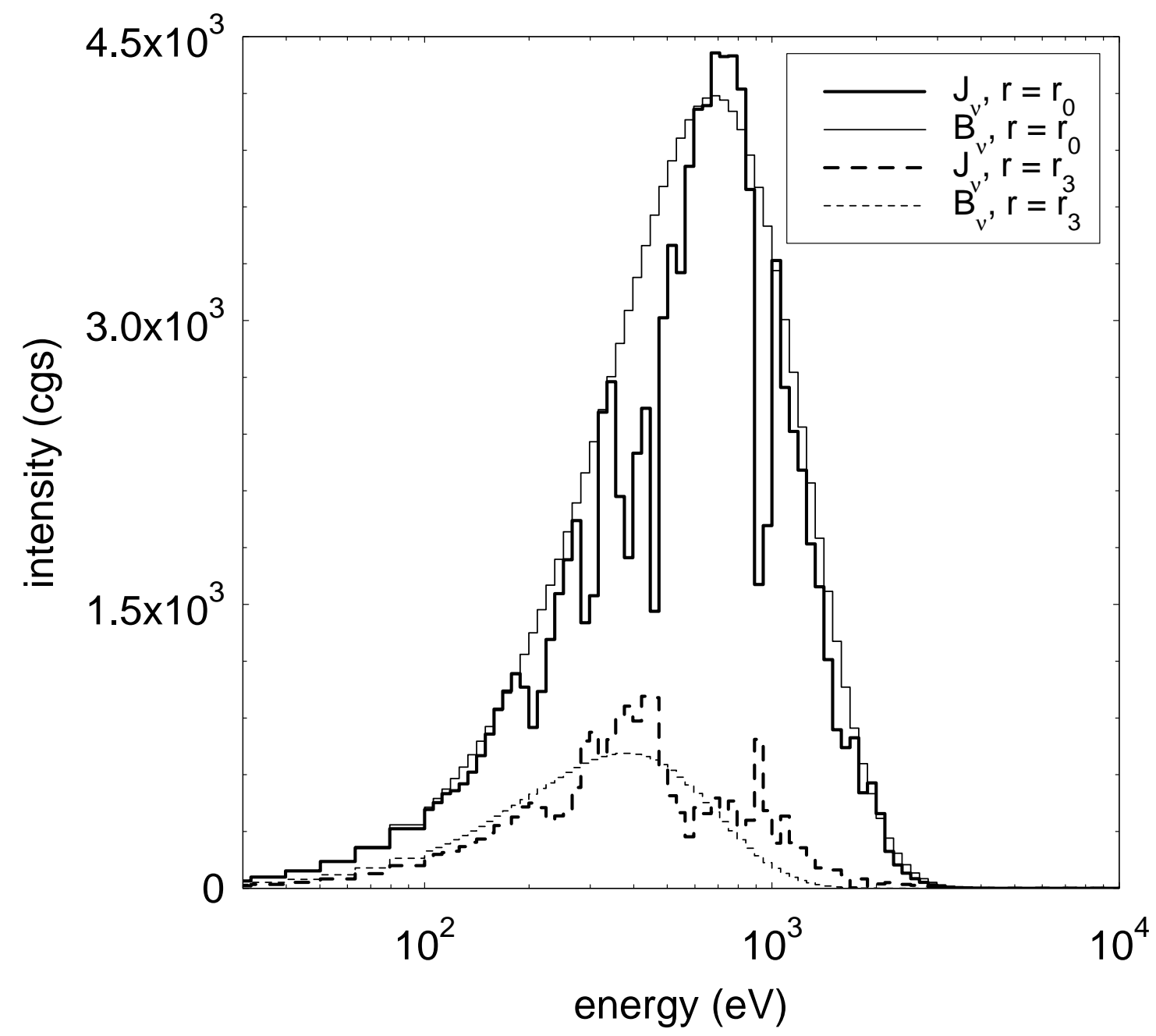

Figure 4b 


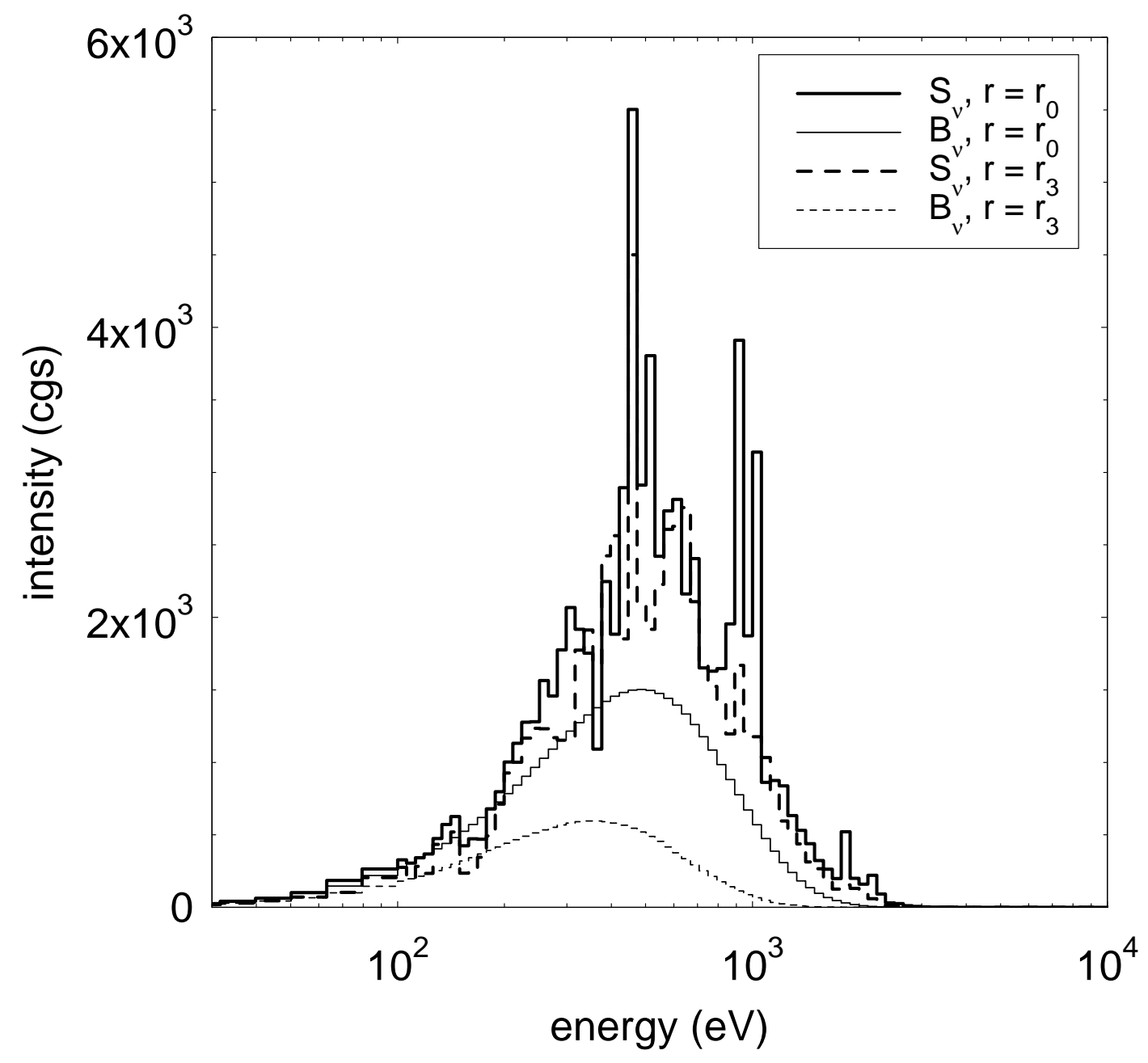

Figure 5a 


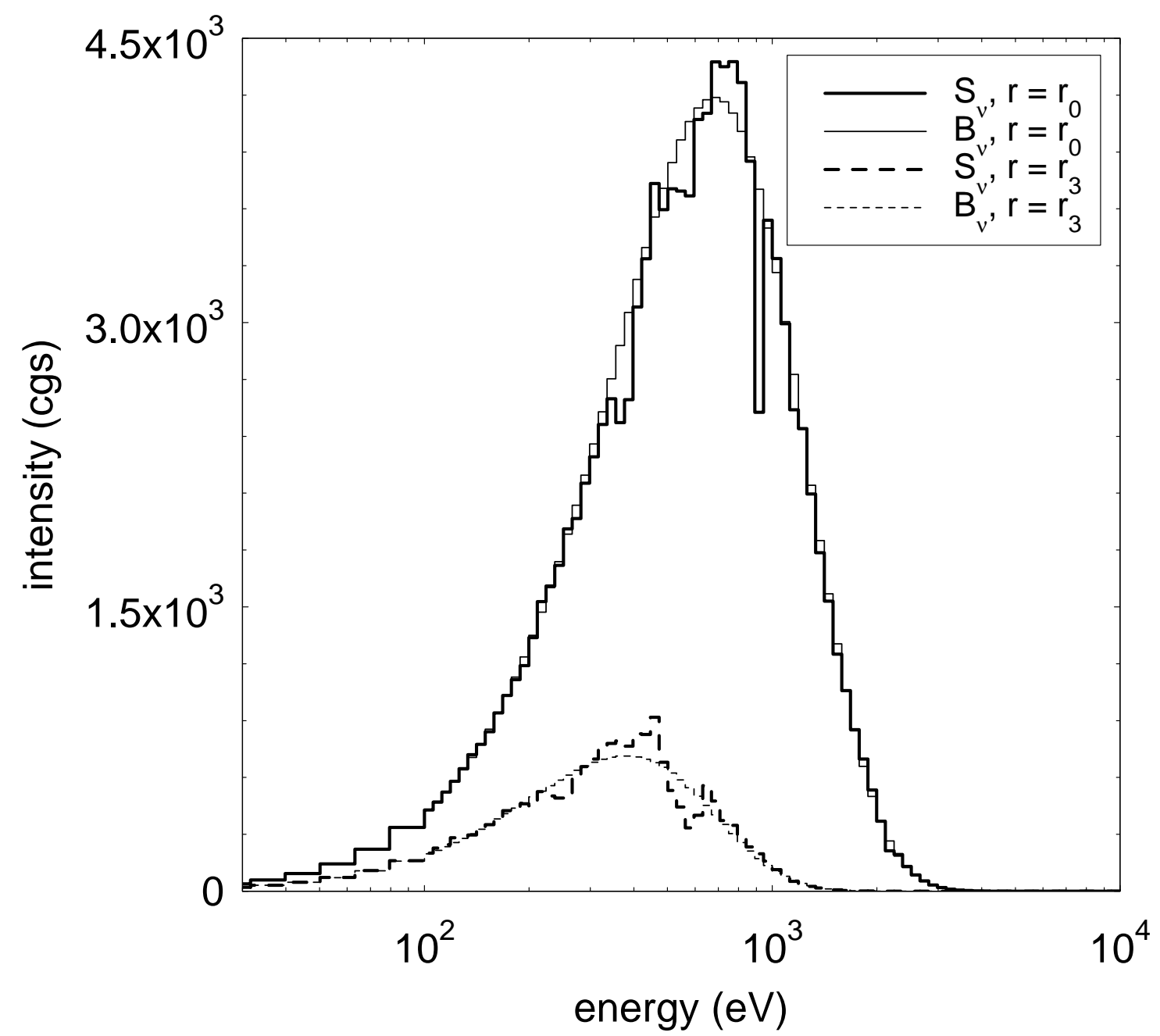

Figure 5b 


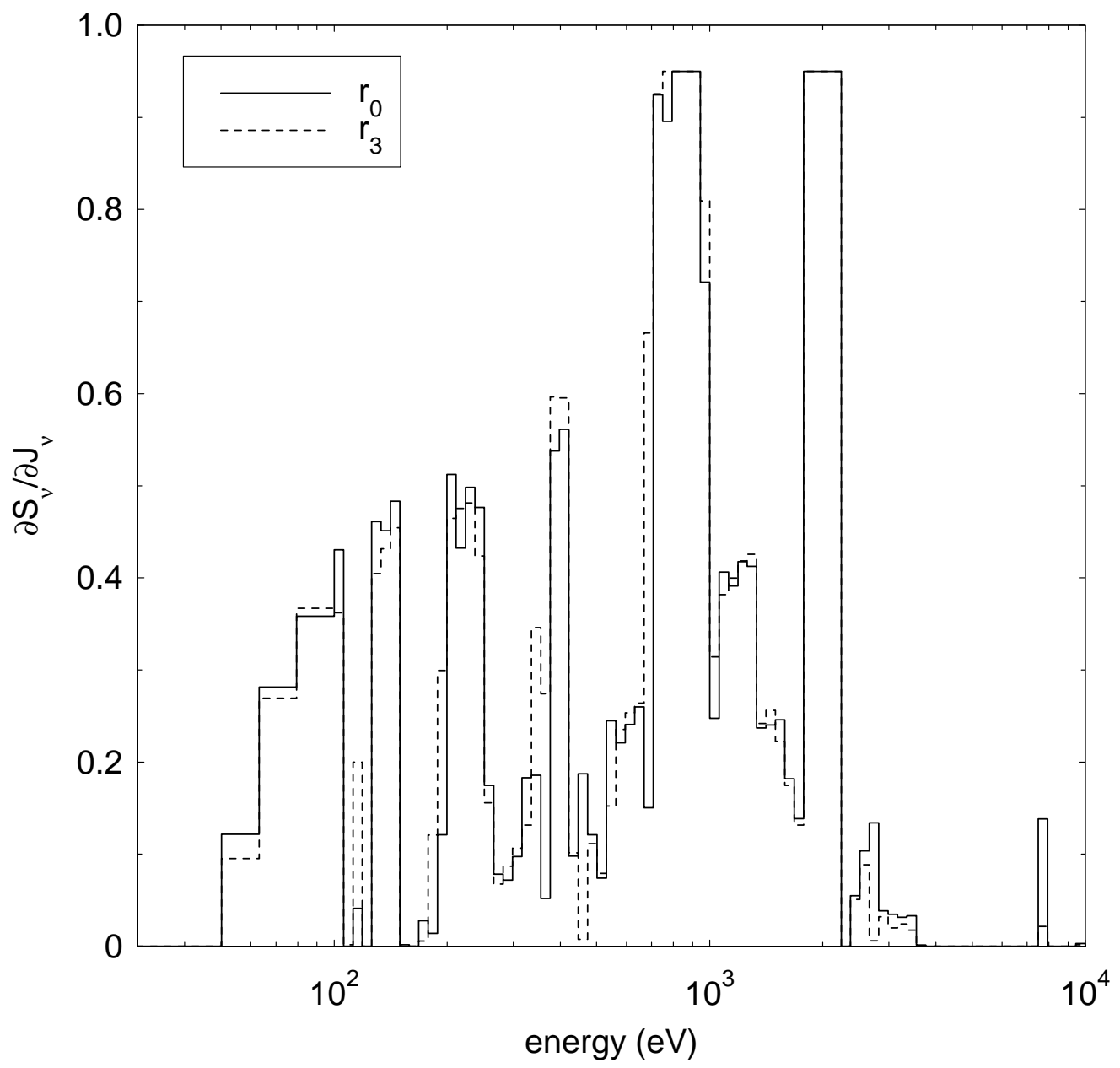

Figure 6a 


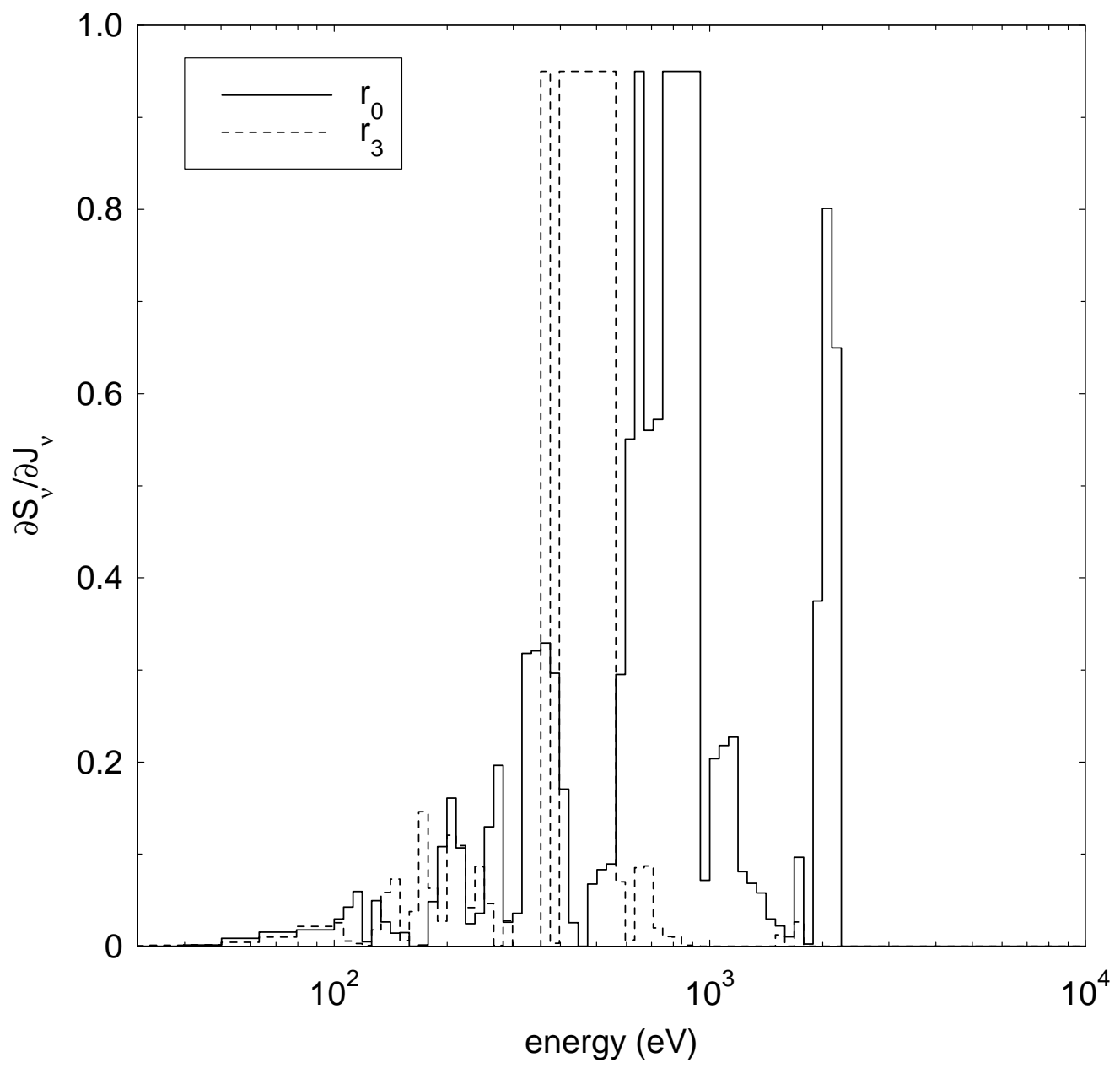

Figure 6b 


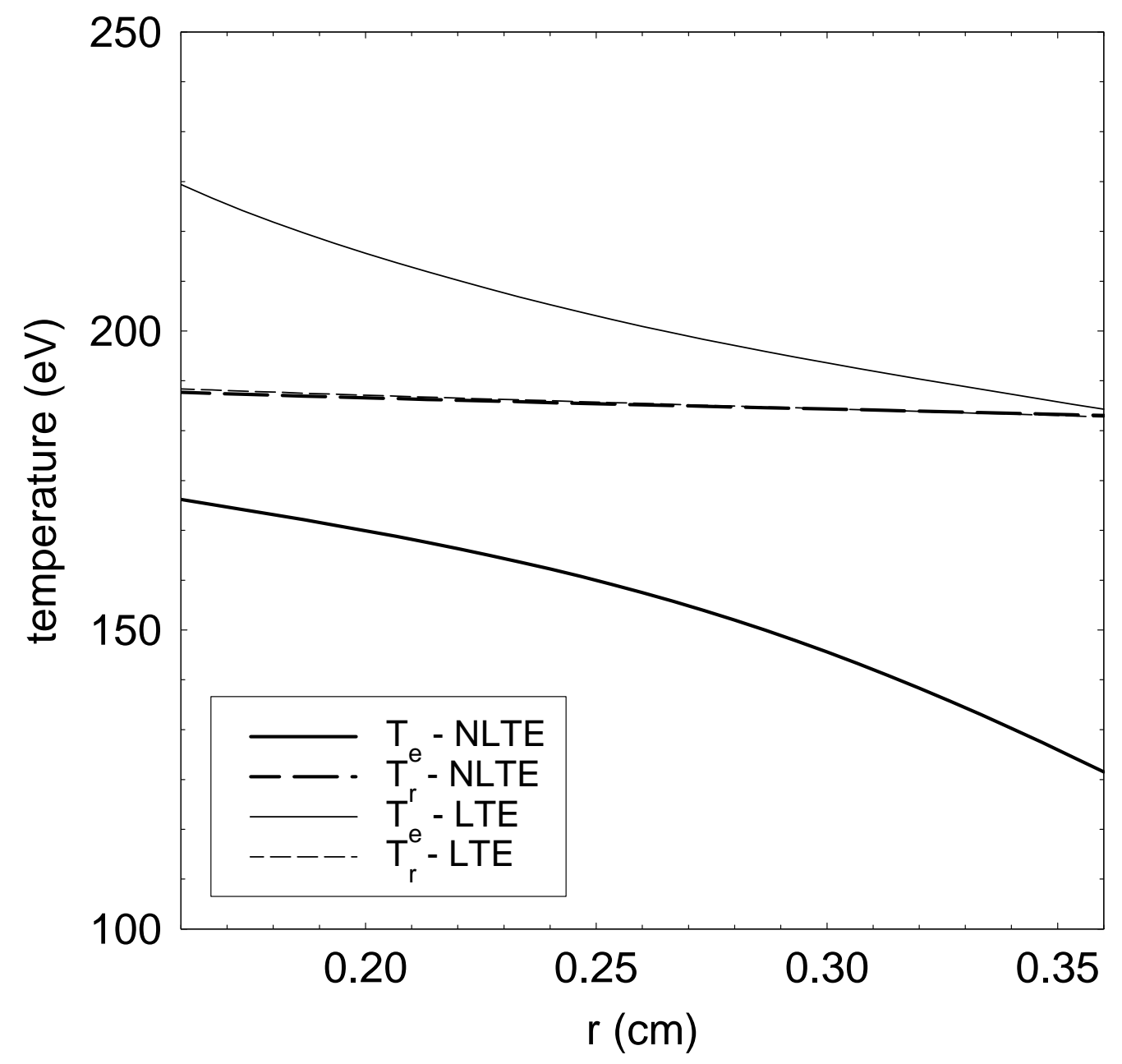

Figure 7a 


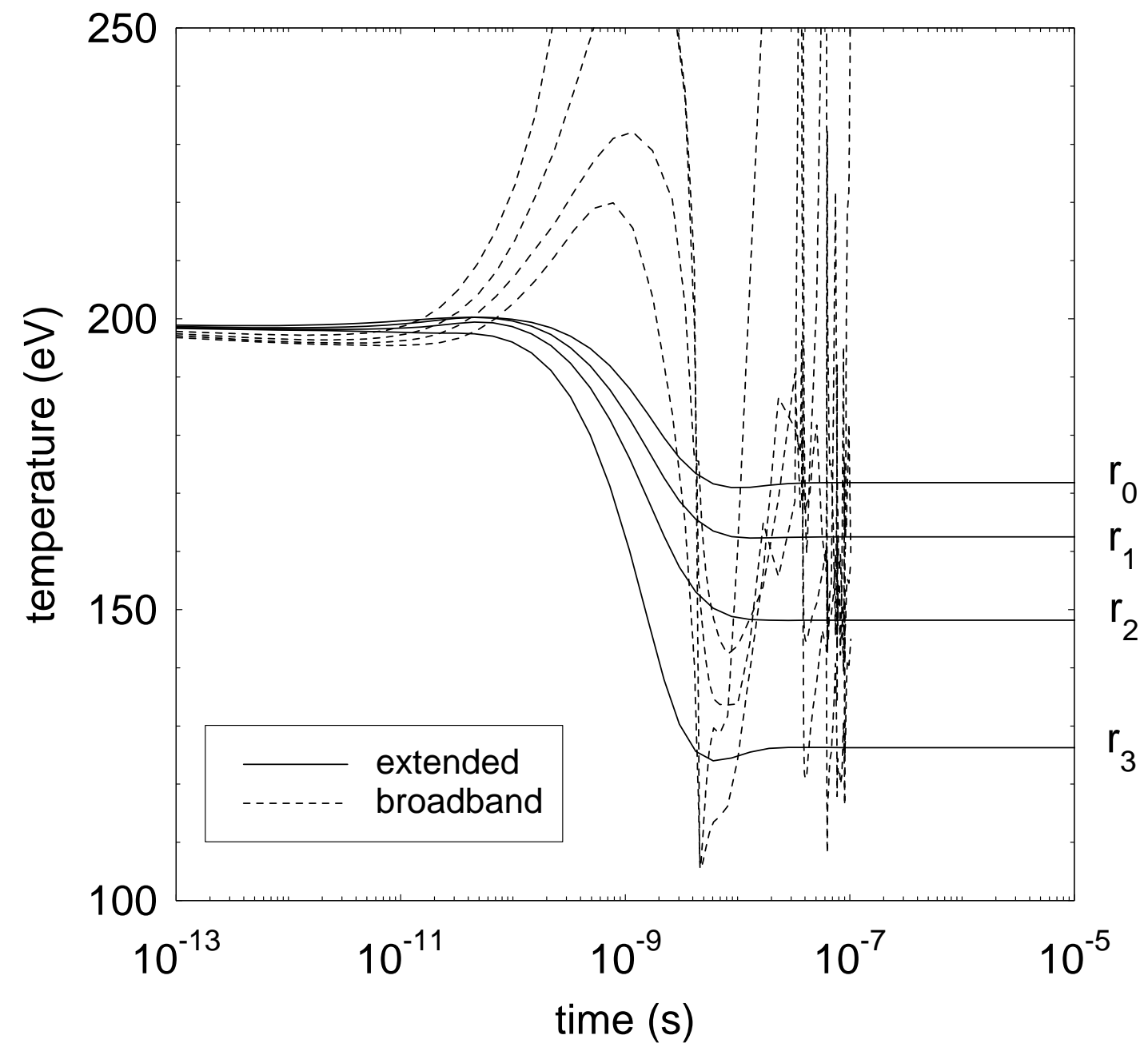

Figure 7b 


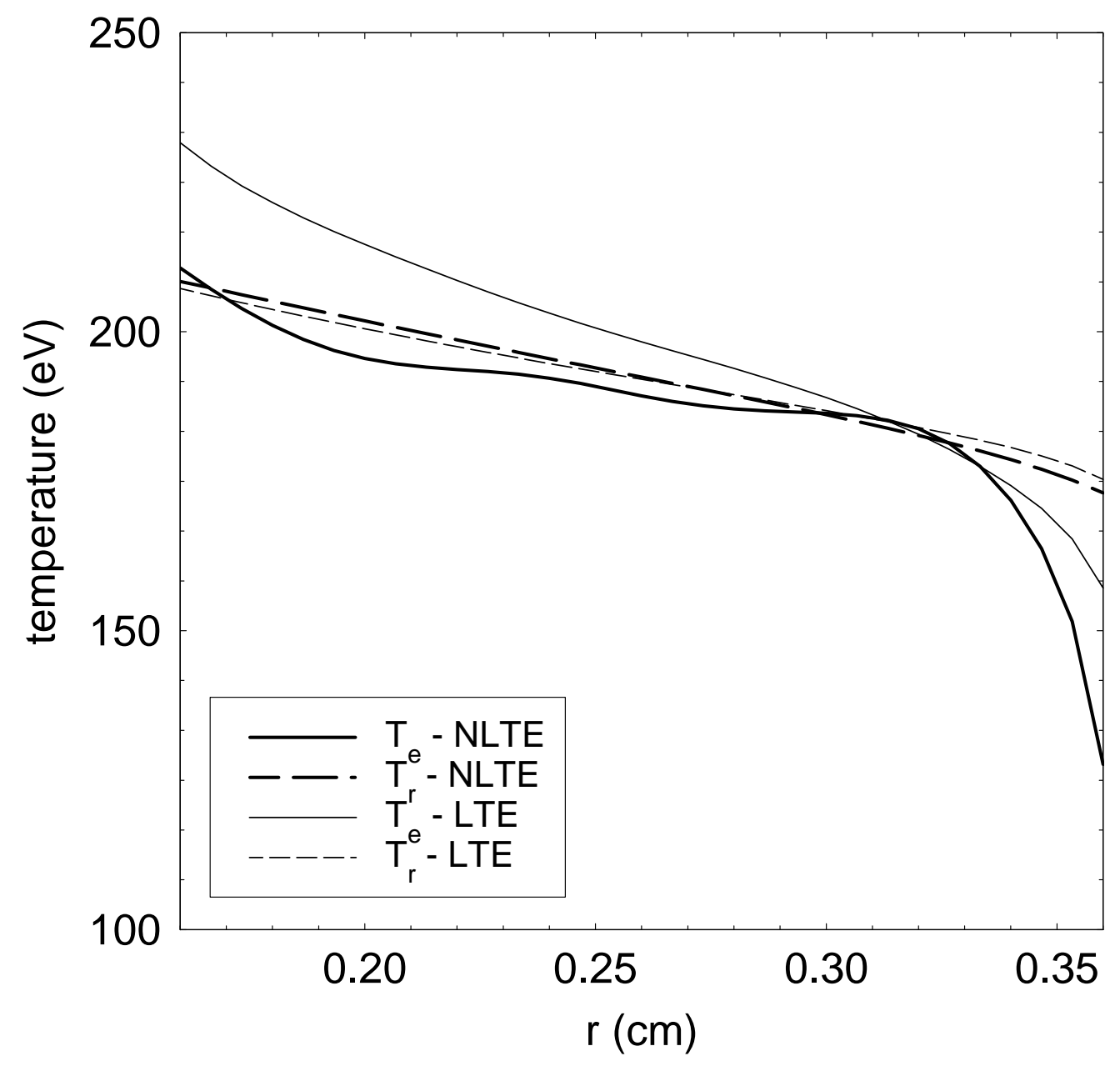

Figure 8a 


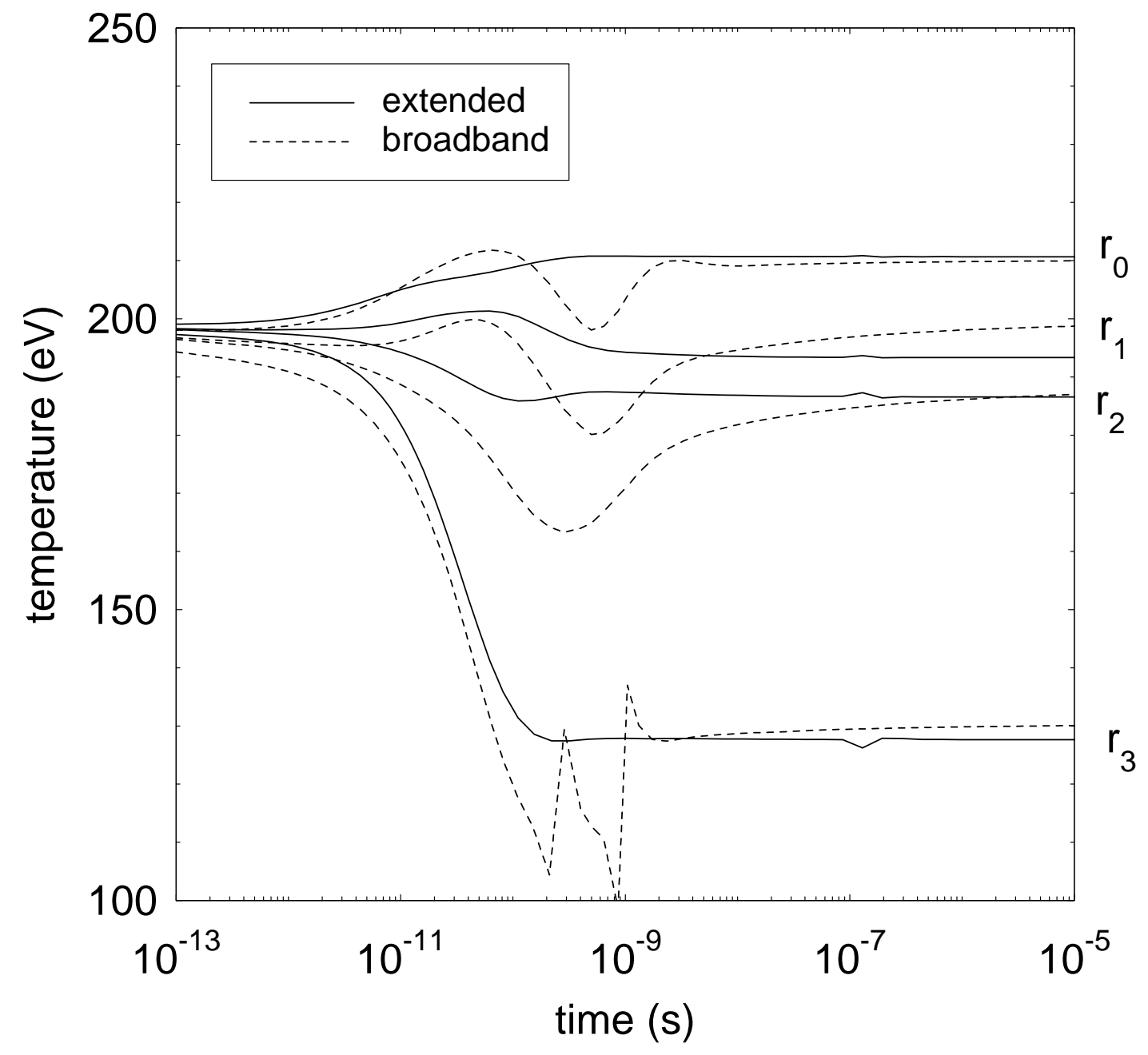

Figure 8b 


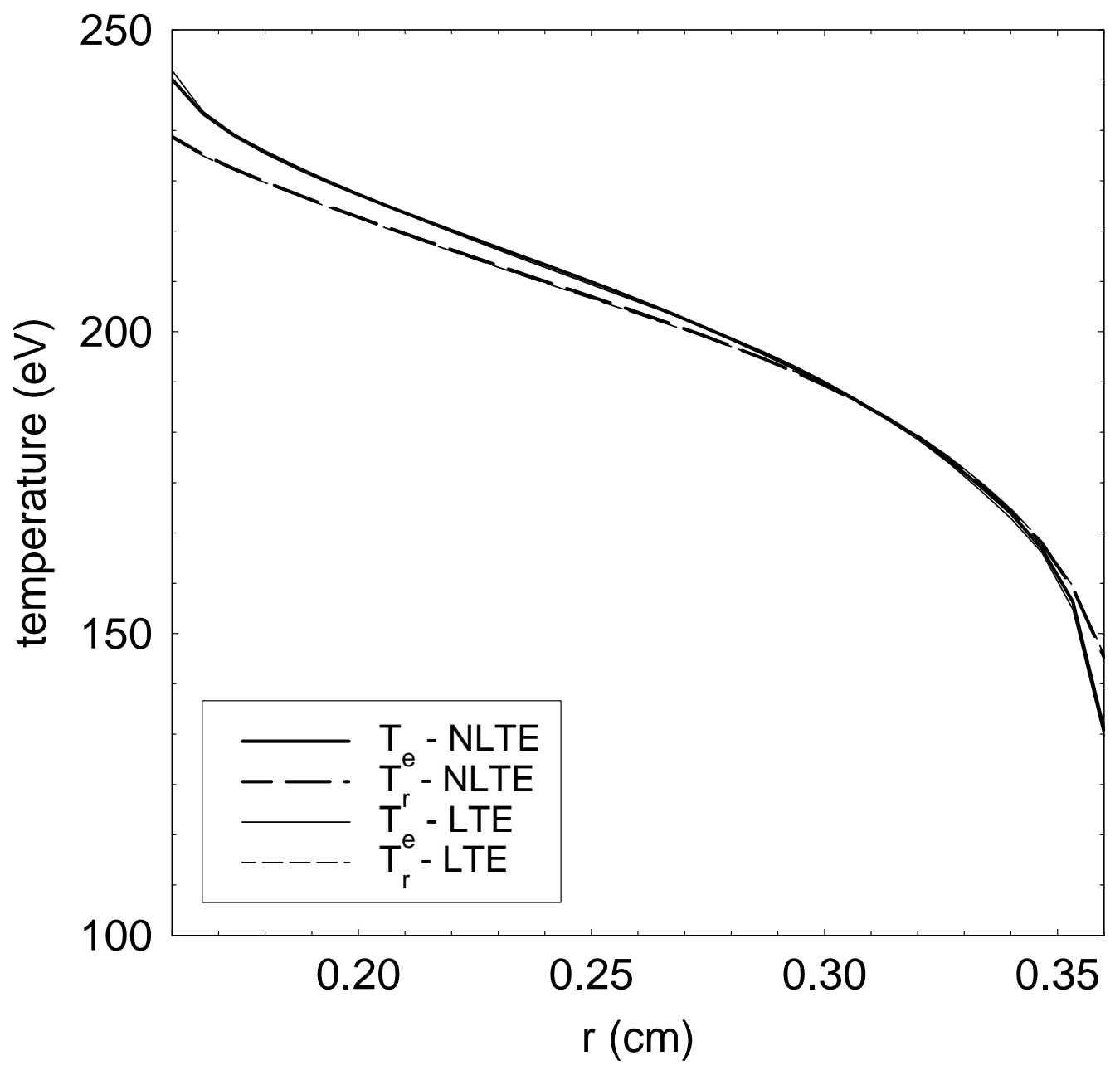

Figure 9a 


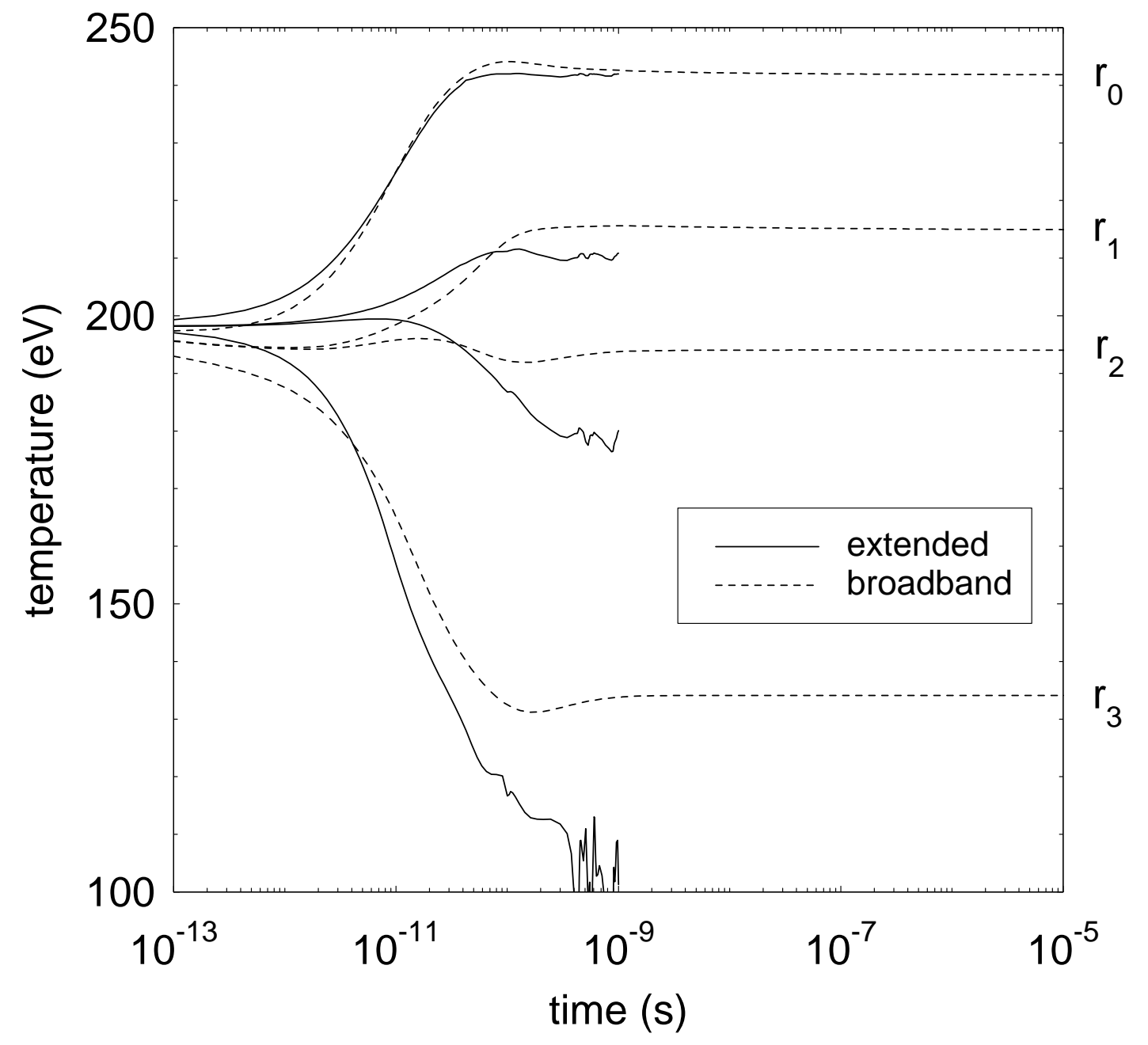

Figure 9b 


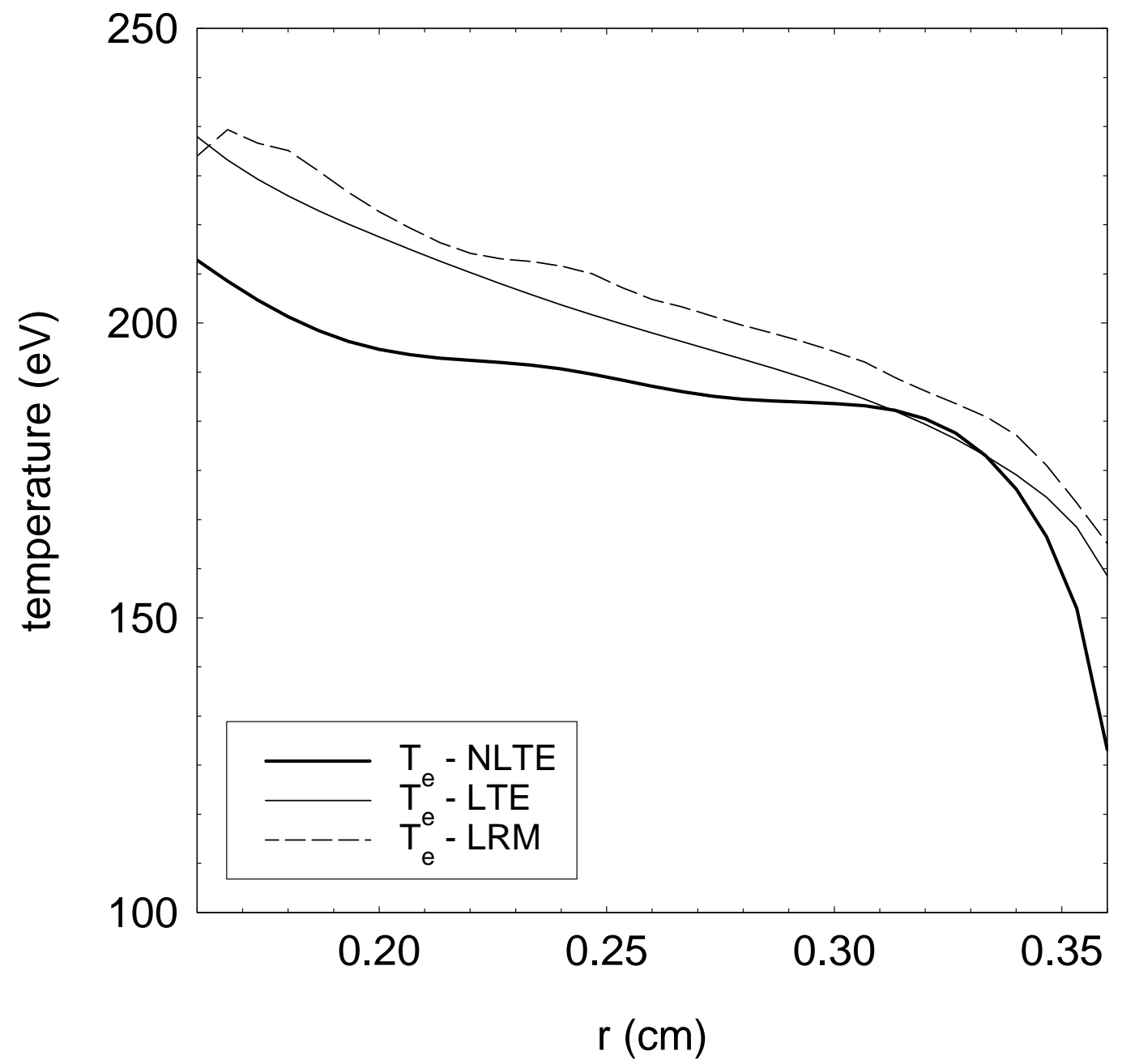

Figure 10 